\title{
BOUNDARY VALUE PROBLEMS FOR ELLIPTIC EQUATIONS
}

\author{
CARLOS E. KENIG*
}

In this note I will describe some recent results, obtained jointly with $R$. Fefferman and J. Pipher [RF-K-P], on the Dirichlet problem for second-order, divergence form elliptic equations, and some work in progress with J. Pipher [K-P] on the corresponding results for the Neumann and regularity problems.

Let us start by recalling some classical results for the Laplacian $\Delta=\sum_{j=1}^{n} \frac{\partial^{2}}{\partial x_{j}^{2}}$ in the unit ball $B=\left\{X=\left(x_{1}, \ldots, x_{n}\right)|| X \mid<1\right\}$. The Dirichlet problem is to solve

$$
\left\{\begin{array}{l}
\Delta u=0 \text { in } B \\
\left.u\right|_{\partial B}=f
\end{array}\right.
$$

while the Neumann problem is to solve

$$
\left\{\begin{array}{l}
\Delta u=0 \text { in } B \\
\left.\frac{\partial u}{\partial N}\right|_{\partial B}=f, \quad \int_{\partial B} f d \sigma=0,
\end{array}\right.
$$

where $\frac{\partial}{\partial N}$ denotes differentiation in the direction of the normal to $\partial B$. As is well known, there are explicit formulas for the solutions of the above problems, and one can then give a very careful analysis of the solutions when, say $f \in L^{p}(\partial B, d \sigma), 1<p<\infty$. In both cases, the boundary values are taken in the sense of non-tangential convergence, i.e., if $Q \in \partial B$, and $\Gamma(Q)=$ $\Gamma_{\alpha}(Q)=\{X \in B|| X-Q \mid<(1+\alpha) \operatorname{dist}(X, \partial B)\}, \alpha>0$, in the case of (D) we have $\lim _{X \rightarrow Q} u(X)=f(Q)$ for a.e. $Q(d \sigma)$, and in the case of (N) $x \in \bar{R}(Q)$

we have $\lim _{X \rightarrow Q} \nabla u(X) N_{Q}=f(Q)$ for a.e. $Q(d \sigma)$. Moreover, the Hardy$x \in \Gamma(Q)$

Littlewood maximal theoren, and in the case of $(N)$, this theorem and the Calderón-Zygmund theory of singular integrals give, denoting by $u^{*}(Q)=$ $\sup _{X \in \Gamma(Q)}|u(X)|$, the estimates

$$
\begin{aligned}
\left\|u^{*}\right\|_{L^{D}(\partial B, d \sigma)} \leq C_{p}\|f\|_{L p(\partial B, d \sigma)} & 1<p \leq \infty \text { for }(\mathrm{D}) \text { and } \\
\left\|(\nabla u)^{*}\right\|_{L^{p}(\partial B, d \sigma)} \leq C_{p}\|f\|_{L^{p}(\partial I, d \sigma)} & 1<p<\infty \text { for }(\mathrm{N})
\end{aligned}
$$

\footnotetext{
*Supported in part by the N.S.F.
} 
so that the non-tangential convergence is in fact "dominated" in the sense of Lebesgue's dominated convergence theorem.

Finally, the regularity problem (R) is the Dirichlet problem (D), but where the boundary data $f \in L_{\uparrow}^{p}(\partial B, d \sigma)$, i.c., it has tangential derivatives, which belong to $L^{p}(\partial B, d \sigma)$. In this case, the Hardy-Littlewood maximal theorem, and the Calderón-Zygmund theory give the estimate

$$
\left\|(\nabla u)^{*}\right\|_{L \cdot p(\partial B, d \sigma)} \leq C_{p}\|f\|_{L_{1}^{p}(\partial B, d \sigma)} \quad 1<p<\infty
$$

In [RF-K-P] and [K-P] we considered elliptic operators $L=\frac{\partial}{\partial x_{i}} a_{i j}(X) \frac{\partial}{\partial x_{j}}$, where $A(X)=\left(a_{i j}(x)\right)$ is a real, symmetric matrix which is bounded, i.e., $\|A\|_{L^{\infty}\left(\mathbb{W}^{n}\right)} \leq \lambda^{-1}$ and elliptic, i.e.,

$$
\langle A(X) \xi, \xi\rangle \geq \lambda|\xi|^{2} \quad \forall \xi \in \mathbb{R}^{n}
$$

where $\lambda>0$. The purpose of these investigations was to understand to what extent the above results extended to

$\left(\mathrm{D}_{L}\right)_{p_{\mathrm{0}}}$

$$
\left\{\begin{array}{l}
L u=0 \text { in } B \\
\left.u\right|_{\partial B}=f \in L^{p_{0}}(\partial B, d \sigma)
\end{array}\right.
$$

$\left(\mathrm{N}_{L}\right)_{p_{0}}$

$$
\left\{\begin{array}{l}
L u=0 \text { in } B \\
\left.\frac{\partial u}{\partial v}\right|_{\partial B}=\left.\langle A \nabla u, N\rangle\right|_{\partial B}=f \in L^{p o}(\partial B, d \sigma), \quad \int_{\partial B} f=0
\end{array}\right.
$$

and $\left(R_{L}\right)_{p_{0}}$, which is the Dirichlet problem for $L$ with data in $L_{1}^{p_{0}}(\partial B, d \sigma)$.

The operators $L$ is described above have been extensively studied, an important results were obtained in the pioneering work of DeGiorgi [DeG], Nash [N], Moser [Mol, Stampacchia [S], and Littman-Stampacchia and Weinberger [L-S-W].

In erder to give some perspective into the understanding of the problems mentionsed before, it is worthwhile to consider $(\mathrm{D})_{p_{0}},(\mathrm{~N})_{p_{0}}$ and $(\mathrm{R})_{p_{0}}$, for the Laplaciant $\Delta$, on a bounded Lipschitz domain $\Omega \subset \mathbb{R}^{n}$, i.e., on domains given lex:ally is $\{X=(x, y): y>\varphi(x)\}, \varphi: \mathbb{R}^{n-1} \rightarrow \mathbf{R},\left|\varphi(x)-\varphi\left(x^{\prime}\right)\right| \leq M\left|x-x^{\prime}\right|$. Such dematins verify (and in fact are characterized by) uniform interior and extorior sons: conditions, and hence the study of non-tangential convergence is meaningful. Morrszer, they have a tangent plane at almost every boundary print, and harce nomal and tangential differentiation make sense. The above preblems are very well mulerstoon in this context, and we have the following resiit.

Theorem. There axists $\varepsilon=\varepsilon(\Omega)>0$ such that

(a) $(D)_{p_{0}}$ holds for $2-\varepsilon<p_{0} \leq \infty$, i.e., if u solves $(D)$, then $\left\|u^{*}\right\|_{L^{p_{0}}(\partial \Omega, d \sigma)}$ $\leq C^{\prime}\|f\|_{L, p o(o n, d \sigma)}$ (Dahiberg [D1]). 
(b) $(N)_{p_{\theta}}$ holds for $1<p_{0}<2+\varepsilon$, i.e.,

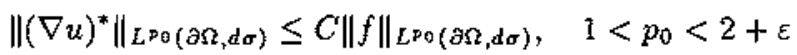

(Jerison-Kenig $[\mathrm{J}-\mathrm{K} 1]$ ) for $p_{0}=2$, Dahlberg-Kenig [D-K] for the general case).

(c) $(R)_{p_{0}}$ holds for $1<p_{0}<2+\varepsilon$, i.e., if $u$ solves the Dirichlet problem with data in $L_{1}^{p o}(\partial \Omega)$, then

$$
\left\|(\nabla u)^{*}\right\|_{L^{p 0}(\partial \Omega, d \sigma)} \leq C\|f\|_{L^{p o}(\partial \Omega),} \quad 1<p_{0}<2+\epsilon
$$

(Jerison-Kenig [J-K1] for $p_{0}=2$, Verchota [V] for the general case, also, Dahlberg-Kenig [D-K] gave a new proof of the general case).

Moreover, in (a), (b), and (c) the range of $p_{0}$ 's is sharp, as simple examples show.

The connection of the above results with our problems comes from the fact that if we make the change of variables $(x, y) \mapsto(x, y-\varphi(x))$, the domain $\Omega$ is mapped to the upper half-plane, but the Laplacian is mapped to an operator $L$ whose coefficients depend on the Jacobian of the change of variables, and hence are bounded measurable, but not any more regular. It is easy to see that $L$ is nevertheless, elliptic.

Let me begin by describing the results for the Dirichlet problem, which are the best understood ones. First, recall that Littman, Stampacchia and Weiberger [L-S-W] showed that the classical Dirichlet problem for $L$ (i.e., with data $f \in C(\partial \Omega)$ and solution $u$ in $C(\bar{\Omega})$ ) has a solutions in $\Omega$ if and only if it has a solution for $\Delta$ in $\Omega$. In particular, this can be done in $B$. Thus, the mapping $f \mapsto u(X)$, where $u$ is the solution of the classical Dirichlet problem with data $f$, for $L$ in $B$ defines a continuous, positive linear functional on $C(\partial B)$, and hence, there exists a probability measure $d \omega_{L}^{X}$ such that $u(X)=\int_{\partial B} f d \omega_{L}^{X}$. $\left\{d \omega_{L}^{X}\right\}$ is called $L$-harmonic measure. The members of this family of measures are mutually absolutely continuous by Moser's [Mo] Harnack principle. We sometimes call $\omega_{L}=\omega_{L}^{0}$ the $L$-harmonic measure. In [C-F-M-S], Caffarelli, Fabes, Mortola and Salsa established an analogue of the classical theorem of Fatou, for $L$. They showed that if $L u=0$ in $B, u \geq 0$, then $u$ has finite non-tangential limits at a.e. $Q \in \partial B\left(d \omega_{L}\right)$. This generalized the corresponding result for harmonic functions on Lipschitz domains, obtained by Hunt and Wheeden $[\mathrm{H}-\mathbf{W}]$. The basic estimates obtained in [C-F-M-S] to prove this Fatou theorem were:

(1) $\omega_{L}\left(\Delta_{2 r}\right) \leq C \omega_{L}\left(\Delta_{r}\right)$ (doubling), where $\Delta_{r}=B(Q, r) \cap \partial B, Q \in \partial B$

(2) If $f \geq 0$, and $u$ solves the Dirichlet problem for $L$ with data $f$, then

$$
u^{*}(Q) \simeq M_{\omega_{L}}(f)(Q) \text {, }
$$

where

$$
M_{\omega_{L}}(f)(Q)=\sup _{\Delta_{r} \ni Q} \frac{1}{\omega_{L}\left(\Delta_{r}\right)} \int_{\Delta_{r}} f d \omega_{L}
$$


Thus, a natural question is whether $\omega_{L}$ and $\sigma$ are mutually absolntely continuous, and also whether $\omega_{L} \in A_{\infty}(d \sigma)$, where $A_{\infty}$ is the classical class of weights introduced by Muckenhoupt [Mu], and Coifman and Fefferman [Co-F]. From (1) and (2) it is easy to see that

Theorem 1. The following are equivalent

(i) $\omega_{L} \in A_{\infty}(d \sigma)$

(ii) $\left(D_{L}\right)_{p_{0}}$ holds for some $p_{0}>1$, i.e,

$$
\left\|u^{*}\right\|_{L, P O(\partial B, d \sigma)} \leq C\|f\|_{L^{p O}(\partial B, d \sigma)}
$$

(iii) $k_{L}=\frac{d w_{L}}{d \sigma}$ exists and belongs to $B_{q_{0}}(d \sigma)$, where $\frac{1}{p_{0}}+\frac{1}{q_{0}}=1$, i.e.,

$$
\left(\frac{1}{\sigma\left(\Delta_{r}\right)} \int_{\Delta_{r}} k_{L}^{q_{0}} d \sigma\right)^{1 / q_{0}} \leq C\left(\frac{1}{\sigma\left(\Delta_{r}\right)} \int_{\Delta_{r}} k_{L} d \sigma\right)
$$

Thus we would like to know when any of the equivalent conditions in Theorem 1 are verified.

In 1981, Caffarelli, Fabes and Kenig [C-F-K], and independently, Modica. and Mortola [M-M], found examples of operators $L$, with continuous coeffcients, such that $\omega_{L}$ and $\sigma$ are mutually singular, and hence for which none of the conditions in Theorem 1 hold. The examples in [C-F-K] were based on the work of Beurling-Ahlfors [B-A] on quasi-conformal mappings. The natural question is what is better about the $L$ 's that arise from Lipschitz domains. The point is that the Jacobian of the change of variables $(x, y) \mapsto(x, y-\varphi(x))$ is coristant in $y$, and $y$ is the normal direction. In fact, in [J-K1], Jerison and Kenig show that if $A(X)$ is $C^{1}$ in the radial direction near $\partial B$, then $\left(D_{L}\right)_{2}$ holds. In light of this, the search was for the "sharp" smoothness condition in the radial variable, at the boundary, which guarantees $\left(D_{L}\right)_{p_{0}}$ for some $p_{0}>1$. All the subsequent work was inspired by classical results in the theory of differentiation of functions (see $[\mathbf{R F}-\mathbf{K}-\mathrm{P}]$ to understand that the analogy is in fact precise).

Let $g: \mathbb{R} \rightarrow \mathbb{R}$ be a measurable function. When is $g$ differentiable a.e.? The necessary condition

$$
|g(x+t)+g(x-t)-2 g(x)|=O(|t|)
$$

is not sufficient, as the Weierstrass nowhere differentiable function shows. On the other hand, if $g$ is differentiable at $x$, the expression on the left in (3) is in fact $o(|t|)$. Thus, we may ask, when is $g$ differentiable a.e., if it satisfics

$$
|g(x+t)+g(x-t)-2 g(x)| \leq|t| \eta(t)
$$


where $\eta(t)$ verifies $\eta(0)=0, \eta$ continuous, $\eta \downarrow$. The answer is provided in the work of Calderón-Zygmund [C-Z], John-Nirenberg [Jo-N], and Stein-Zygmund [St-Z]: if $\int_{0} \eta^{2}(t) \frac{d t}{t}<\infty, g^{t}$ exists a.e. and is in $L_{\mathrm{foc}}^{p}$ for all $p<\infty$. Moreover, given such an $\eta$, with $\int_{0} \eta^{2}(t) \frac{d t}{t}=+\infty$, there exists a $g$ verifying (4) which is nowhere differentiable.

A sharper result, which is necessary and sufficient for differentiability for a given $g$, an which involves integral conditions instead of the pointwise condition (4) is due to Marcinkiewicz [M], and Stein-Zygmund [St-Z]. They showed that, under the necessary condition (3), a necessary and sufficient condition for the a.e. differentiability of $g$ is

$$
\int_{|t|<1} \frac{|g(x+t)+g(x-t)-2 g(x)|^{2}}{|t|^{2}} \frac{d t}{|t|}<\infty \text { for a.e. } x .
$$

In our analogy, we think of (3) as an "additive" analogue of the "multiplicative" condition (1), and scek the corresponding analogues of (4) and (5). See [RF-K-P] for the details of this argument.

Continuing with our description of further developments, in [F-J-K], Fabes, Jerison and Kenig showed that if $A \in C(\bar{B})$ and

$$
\eta(t)=\sup _{\substack{Q<s<t \\|Q|=1}}|A((I-s) Q)-A(Q)|
$$

verifies $\int_{0} \eta^{2}(t) d t / t<\infty$, then $\left(D_{L}\right)_{p_{0}}$ was valid for every $1<p_{0}<\infty$. Moreover, the counterexamples in [C-F-K] showed that given any $\eta$ with $\eta(0)=0, \eta \downarrow$, and $\int_{0} \eta^{2}(t) d t / t=+\infty$, we could find $A \in C(\bar{B})$, as above, with $\omega_{L}$ and $\sigma$ mutually singular.

In [D3], B.E.J. Dahlberg extended this result, with a condition in the spirit of (5), and which extended the Lipschitz domain result. He introduced the quantities

$$
\varepsilon(X)=\sup _{Y \in B(X, \delta(X) / 2)}|A(Y)-A(Y /|Y|)|
$$

and

$$
h(r, Q)=\left(\frac{1}{r^{n-1}} \iint_{B(Q, r\} \cap a B} \frac{\varepsilon^{2}(X)}{|1-| X||} d X\right)^{1 / 2} \quad 0<r<1, Q \in \partial B
$$

The quantity $h(r, Q)$ expresses an integral way of measuring smoothness in the radial direction, at $\partial B$, in a "dilation invariant" manner. Dahlberg's result was that, if

$$
\varlimsup_{r \rightarrow 0} \sup _{|Q|=1} h(r, Q)=0
$$


then there exists $\varepsilon>0$ such that $\left(D_{L}\right)_{p_{0}}$ holds for $2-\varepsilon<p_{0} \leq \infty$.

If then becomes of interest to investigate to what extent the $\overline{\lim }$ in (8) being 0 was necessary. The first progress in this direction was made by $R$. Fefferman, [RF], who introduced the quantity

$$
A(\varepsilon)(Q)=\left(\iint_{\Gamma(Q)} \frac{\varepsilon^{2}(X)}{\left.|1-| X\right|^{n}} d x\right)^{1 / 2}
$$

Note that $h(r, Q) \leq \frac{C}{r^{n-1}} \int_{B(Q, r) \cap a B} A(\varepsilon)^{2} d \sigma$. R. Fefferman [RF] showed that, if $\|A(\varepsilon)\|_{L^{\infty}(\partial B)} \leq C$, then $\omega_{L} \in A_{\infty}(d \sigma)$ and hence $\left(\mathrm{D}_{L}\right)_{p_{0}}$ holds for some $p_{0}>1$. In [RF-K-P], R. Fefferman, Kenig and Pipher prove the following

\section{Theorem 2.}

(i) If $\sup _{0<r<1} \sup _{|Q|=1} h(r, Q)<+\infty$, then $\omega_{L} \in A_{\infty}(d \sigma)$, and hence $\left(D_{L}\right)_{p_{0}}$ holds for some $p_{0}>1$.

(ii) If $E \subset \partial B$ is closed, and $A(\varepsilon)(Q)<+\infty$ for each $Q \in E$, then $\omega_{L}$ and $\sigma$ are mutually absolutely continuous on $E$.

This theorem is sharp in a number of ways.

Theorem 3. If $n=2$, and $\omega \in A_{\infty}(d \sigma)$, there exists $A(X)$ such that $A(Q)=$ I for $Q \in \partial B$, and such that $\omega_{L} \simeq \omega$ and $\sup _{0<r<1} \sup _{|Q|=1} h(r, Q)<\infty$, i.e., every $A_{\infty}$ measure arises in the manner described by Theorem 2.

Theorem 4. If $n=2$, and $a(X)$ is any bounded function on $B$, which is "slowly varying" (see [RF-K-P] for the precise definition) such that

$$
\left(\sup _{0<r<1} \sup _{|Q|=1} \frac{1}{r^{n-1}} \iint_{B(Q, r) \cap B} \frac{a^{2}(X)}{11-|X| \mid} d X\right)^{1 / r}=+\infty
$$

we can find $A(X)$ with $A(Q)=I$ for all $Q \in \partial B$, with

$$
\frac{1}{r^{n-1}} \iint_{B(Q, r) \cap B} \frac{\varepsilon^{2}(X)}{|1-| X||} d X \leq C\left\{\frac{1}{r^{n-1}} \iint_{B(Q, 2 r) \cap B} \frac{a^{2}(X)}{|1-| X||} d X+1\right\}
$$

for all $0<r<1 / 4, Q \in \partial B$, and such that $\omega_{L} \notin A_{\infty}(d \sigma)$, i.e., the Carleson measure condition $\sup _{0<r<1} \sup _{|Q|=1} h(r, Q)<+\infty$ is the weakest condition of its kind that guarantees that $\omega_{L} \in A_{\infty}(d \sigma)$.

To establish Theorems 3 and 4 we use the Beurling-Ahlfors results, as in [C-F-K], together with some new characterizations of $A_{\infty}$ weights. (See [RFK-P] for the details.) 
I will now turn my attention to work in progress [K-P], with J. Pipher on the regularity and Neumann problems.

First, notice that in the context that we are working in, $(\nabla u)^{*}$ may be identically infinite, even if $A(X)=A(X /|X|)$. This is because $\nabla u$ need not have pointwise values in the interior, when there is no further regularity on the coefficients. The most that can be said in general is that it is in $L_{\text {loc }}^{2}(B)$. Thus, we need a new definition

$$
(\nabla u)^{* *}(Q)=\sup _{X \in \Gamma(Q)}\left(\frac{1}{\left.|1-| X\right|^{n}} \iint_{B(X,(1-|X|) \alpha / 4)}|\nabla u(Y)|^{2} d Y\right)^{1 / 2}
$$

Note that when we are working with the Laplacian on a Lipschitz domain, $(\nabla u)^{* *}$ and $(\nabla u)^{*}$ are comparable, if we use wider cones.

Moreover, the examples in [C-F-K] can be used, in conjunction with well known properties on the boundedness of the Hilbert transform with weights ([H-M-W]) to show that there exists $L$ with continuous coefficients such that the estimates $\left(\mathrm{N}_{L}\right)_{p_{0}}$ and $\left(\mathrm{R}_{L}\right)_{p_{0}}$ :

$$
\left\|(\nabla u)^{* *}\right\|_{L, P \circ(\partial B, d \sigma)} \leq C\|f\|_{L^{D O}(\partial B, d \sigma)} \quad f=\left.\frac{\partial u}{\partial v}\right|_{\partial B}
$$

and

$$
\left\|(\nabla u)^{* *}\right\|_{L^{p_{O}(\partial B, d \sigma)}} \leq C\|f\|_{L^{p O}(\partial B)},\left.\quad u\right|_{\partial B}=f,
$$

hold for no $p, 1 \leq p_{0} \leq \infty$.

We have been able to establish the following positive result:

Theorem 5. Assume that (8) holds. Then, there exists $\varepsilon>0$ such that, for $1<p_{0}<2+\varepsilon,\left(N_{L}\right)_{p_{0}}$ and $\left(R_{L}\right)_{p_{0}}$ hold.

What happens when merely $\sup _{0<r<1} \sup _{|Q|=1} h(r, Q)<\infty$, in this situation remains an open problem.

\section{References}

[B-A] A. BEuRling AND L. AhlFors, The boundary correspondence under quasi-conformal mappings, Acta Math. 96 (1956), 125-142.

[C-F-K] L. Caffarelli, E. Fabes and C. Kenig, Completely singular elliptic-harmonic measures, Ind. U. Math. 30 (1981), 917-924.

[C-F-M-S] L. Caffarfilli, E. Fabes, S. Mortola and S. Salsa, Boundary behavior of non-negative solutions of elliptic operators in divergence form, Ind. U. Math. 30 (1981), 621-640. 
[C-Z] A.P. CAIDERón AND A. ZYGMUND, Local properties of solutions of elliptic parital differential equations, Studia Math. 20 (1961), 171-225.

[Co-F] R. Coifman AND C. FefFerman, Weighted norm inequalities of maximal functions arid singular integrals, Studia Math. 51 (1974), 241-250.

[D1] B.E.J. Dalhberg, On estimates of harmonic measure, Arch. Rat. Mech. Anal. 65 (1977), 272-288.

[D2] B.E.J. Dalhberg, On the Poisson integral for Lipschitz and $C^{1}$ domain, Studia Math. 66 (1979), 7-24.

[D3] B.E.J. DALHBERG, On the absolute continuity of elliptic measures, Amer. J. Math. 108 (1986), 1119-1138.

[D-K] B.E.J. Dalhberg And C. KeNIG, Hardy spaces and the Neumann problem in $L^{p}$ for Laplace's equation in Lipschitz domains, Annals of $M a t h$. 125 (1987), 437-465.

[DeG] E. DEGIORGI, Sulle differenziabilata e analiticita delle estremali degli integrali multipli regulari, Mem. Acad. Sci. Torino 3 (1957), 25-43.

[F-J-K] E. FABES, D. JERISON AND C. KENIG, Necessary and sufficient conditions for absolute continuity of elliptic harmonic measure, Annals of Math. 119 (1984), 121-141.

[RF] R. FEFFERMAN, A criterion for the absolute continuity of the harmonic measure associated with an elliptic opcrator, A.M.S. 2 (1989), 127-135.

[RF-K-P] R. FefFerman, C. Kenig and J. Pinper, The theory of weights and the Dirichlet problem for elliptic equations, preprint.

[H-M-W] R. HUnT, B. MUCKENhOUPT AND R. WhEEDFN, Weighted norm inequalities for the conjugate function and Hilbert transform, Trans. A.M.S. 176 (1973), 227-251.

[H-W] R. HUNT AND R. WHEEDEN, On the boundary values of harmonic functions, Trans. A.M.S. 132 (1968), 307-322.

[J-K1] D. Jerison and C. Kenig, The Neumann problem on Lipschitz domains, Bull. A.M.S. 4 (1981), 203-207.

[J-K2] D. JERISON AND C. KENIG, The Dirichlet problem in nort-smooth domains, Annals of Math. 113 (1981), 367-382.

[Jo-N] F. John AND L. NiREnBERG, On functions of bounded mean ascillation, Comm. Pure and Appl. Math. 14 (1961), 415-426.

[K-P] C. Kenig and J. Piphen, The Neumann problen for second order elliptic operators, manuscript in preparation.

[L-S-W] W. Littman, G. Stampacchia and H.F. Weinberger, Regular points for elliptic equations with discontinuous coefficients, Ann. Scuola Norm. Pisa 17 (1963), 45-79.

[M] J. Marcinkiewicz, Sur quelques intégrales du type de Dini, $A n n$. Soc. Pol. Math. 17 (1938), 42-50. 
[M-M] L. MOdica AND S. MORTOLA, Construction of a singular elliptic-harmonic measure, Manuscripta Math. 33 (1980), 81-98.

[Mo] J. Moser, On Harnack's theorem for elliptic differential equations, Comm. Pure and Appl. Math. 14 (1961), 577-591.

[Mu] B. Muckenhoupt, The equivalence of two conditions for weights, Siudia Math. 49 (1975), 101-106.

[N] J. NASH, Continuity of the solutions of parabolic and elliptic equations, Amer. J. Math. 80 (1957), 931-954.

[S] G. STAMPACCHIA, "Equations élliptiques du second order à coefficients discontinus," Séminaire Eq. Der. Part., Collège de France, Paris, 1963/64.

[St-Z] E. STEIN AND A. ZYGMUND, On the differentiability of functions, Studia Math. 23 (1964), 247-283.

[V] G. VERCHOTA, Layer potentials and regularity for the Dirichlet problem for Laplace's equation in Lipschitz domains, J. of Funct. Anal. 59 (1984), $572-611$.

Department of Mathematics

University of Chicago

Chicago IL 60637

U.S.A. 